\title{
Special Issue: Game Programming
}

\author{
Tetsuro TAnaka ${ }^{1, a^{2}}$
}

Starting with chess, games have been a very popular research area outside Japan. With the building of strong programs as the prime objective, game research has provided important contributions in a wide variety of research areas such as search, planning, machine learning, specialized hardware and the study of cognitive processes involved in game playing. On the other hand, in Japan traditionally prominent games like shogi and Go have been recognized as the next research targets after chess and are attracting a lot of attention from researchers around the world as well as from researchers inside Japan.

The aim of this special issue is to present a collection of the latest results in game programming research. Relevant topics include the current state of game playing programs, algorithms for solving puzzles, programs for creating puzzles, cognitive processes of game players, learning of game strategic knowledge, video games, online games, applications of game theory regarding the analysis of human behavior, multi-agent games (for example, soccer), the analysis of the history of games using computers, the development of new games using computers, specialized hardware for game playing programs, systems supporting the mastering of games by human players, and any other topics that are related to game programming.

We had sixteen submissions. After the due date of the paper submission, 20th February 2012, we had three editorial meetings, the first one on 28th February 2012, the second one on 1st May 2012, the last one on 17th July 2012. Finally we selected 8 to be included in the special issue after a thorough and rigorous review process.

We thank the authors and the referees for carefull reviews. We also thank the editorial commitee of this journal for approving this specual issue.

\section{The Editorial Committee}

\section{- Editor in-Chief:}

Tetsuro Tanaka (The University of Tokyo)

\section{- Editorial Committee:}

Hiroyuki Iida (Japan Advanced Institute of Science and Technology)

Takeshi Ito (The University of Electro-Communications) Tomoyuki Kaneko (The University of Tokyo)

Akihiro Kishimoto (Tokyo Institute of Technology)

Yoshiyuki Kotani (Tokyo University of Agriculture and

The University of Tokyo, Meguro, Tokyo 153-8902, Japan

a) ktanaka@tanaka.ecc.u-tokyo.ac.jp

\author{
Technology)
}

Nobusuke Sasaki (Prefectural University of Hiroshima)

Makoto Sato (Toshiba)

Masato Shinoda (Nara Women's University)

Takenobu Takizawa (Waseda University)

Yoshimasa Tsuruoka (The University of Tokyo)

Teigo Nakamura (Kyushu Institute of Technology)

Tsuyoshi Hashimoto (Matsue College of Technology)

Hitoshi Matsubara (Future University Hakodate)

Masakazu Muramatsu (The University of ElectroCommunications)

Reijer Grimbergen (Tokyo University of Technology) 